\title{
The Review of Anti-aging Mechanism of Polyphenols on Caenorhabditis elegans
}

\author{
Limin Liu ${ }^{1,2}$, Peisen Guo ${ }^{1}$, Peixi Wang ${ }^{2}$, Shanqing Zheng ${ }^{3}$, Zhi Qu ${ }^{2 *}$ and Nan Liu 1,2,4* \\ ${ }^{1}$ College of Public Health, Zhengzhou University, Zhengzhou, China, ${ }^{2}$ Institute of Chronic Disease Risks Assessment, School \\ of Nursing and Health, Henan University, Kaifeng, China, ${ }^{3}$ School of Basic Medical Sciences, Henan University, Kaifeng, \\ China, ${ }^{4}$ Institute of Environment and Health, South China Hospital, Health Science Center, Shenzhen University, Shenzhen, \\ China
}

\section{OPEN ACCESS}

Edited by:

Miaomiao Yuan,

Sun Yat-Sen University, China

Reviewed by:

Jie Gao,

Guangxi University, China

Rajendran Velmurugan,

Chulalongkorn University, Thailand

*Correspondence:

Zhi Qu

10210056@henu.edu.cn orcid.org/0000-0001-9898-4746

Nan Liu

13688869875@163.com;

LNQ555@126.com orcid.org/0000-0002-8895-3169

Specialty section:

This article was submitted to Synthetic Biology,

a section of the journal Frontiers in Bioengineering and Biotechnology

Received: 30 November 2020 Accepted: 07 June 2021

Published: 01 July 2021

Citation:

Liu L, Guo P, Wang P, Zheng S, Qu Z and Liu N (2021) The Review of Anti-aging Mechanism of Polyphenols on Caenorhabditis elegans. Front. Bioeng. Biotechnol. 9:635768. doi: 10.3389/fbioe.2021.635768
Micronutrients extracted from natural plants or made by biological synthesis are widely used in anti-aging research and applications. Among more than 30 effective anti-aging substances, employing polyphenol organic compounds for modification or delaying of the aging process attracts great interest because of their distinct contribution in the prevention of degenerative diseases, such as cardiovascular disease and cancer. There is a profound potential for polyphenol extracts in the research of aging and the related diseases of the elderly. Previous studies have mainly focused on the properties of polyphenols implicated in free radical scavenging; however, the anti-oxidant effect cannot fully elaborate its biological functions, such as neuroprotection, A $\beta$ protein production, ion channel coupling, and signal transduction pathways. Caenorhabditis elegans (C. elegans) has been considered as an ideal model organism for exploring the mechanism of anti-aging research and is broadly utilized in screening for natural bioactive substances. In this review, we have described the molecular mechanisms and pathways responsible for the slowdown of aging processes exerted by polyphenols. We also have discussed the possible mechanisms for their anti-oxidant and anti-aging properties in C. elegans from the perspective of different classifications of the specific polyphenols, such as flavonols, anthocyanins, flavan-3-ols, hydroxybenzoic acid, hydroxycinnamic acid, and stilbenes.

Keywords: polyphenols, Caenorhabditis elegans, anti-aging, anti-oxidant, insulin/insulin-like signaling pathway

\section{INTRODUCTION}

Aging is considered a universal physiological process that is accompanied by systemic changes in the structural integrity of cells that are caused by alterations in metabolic and signal transduction pathways (Childs et al., 2015). Understanding of the biological mechanisms of aging and longevity has been growing remarkably over the past two decades. At the molecular level, senescence is strongly associated with susceptibility to chronic diseases and disorders, such as chronic fibrosis, severe atherosclerosis, diabetes, osteoarthritis, and ultimately death (Childs et al., 2016; Amor et al., 2020). Among the various anti-aging methods and preventive strategies, the use of micronutrients or biologically active substances is considered a practical and efficient method that targets a variety of intracellular/extracellular pathways (Sahin et al., 2011; Johnson et al., 2013; Li et al., 2017).

Nutrients and bioactive substances have shed new light on the prevention and treatment of chronic diseases and aging. For example, short-term supplementation with appropriate doses of vitamin $\mathrm{C}$ or vitamin $\mathrm{C}$ plus $\mathrm{E}$ has already been confirmed to improve the immunological function 
in the elderly and contribute to health and longevity (De la Fuente et al., 2020). Most of the substances that exert bioactive properties originate from natural plants and animals and have been extensively studied for their preventive and therapeutic effects against chronic diseases and aging. Functional nutrition is of great significance to human health; however, the high cost involved in extracting and purifying bioactive compounds from natural sources in the past has limited the rapid growth of the market. Thus, with the development of synthetic biology technology, a few important functional nutrients can be produced at a low cost by biological manufacturing. In the future, biological manufacturing is expected to be replaced by traditional extraction techniques or functional nutritional chemicals. So far, plant polyphenols, such as blueberry polyphenols, black tea and green tea polyphenols, and tocotrienols in vegetable oils, have been proved to delay the aging process in model organisms (Adachi and Ishii, 2000; Wilson et al., 2006; Peng et al., 2009; Salminen et al., 2012; Zarse et al., 2012). The anti-aging effects of these polyphenols are mostly related to their anti-oxidant properties and their ability to scavenge free radicals. It has been reported that resveratrol, a polyphenol compound in red wine, could slow down aging in Caenorhabditis elegans due to the reduction of mitochondrial respiration (Wood et al., 2004). The understanding of human aging and longevity might be improved by elucidating the molecular mechanism of aging in $C$. elegans (Park et al., 2020).

\section{ADVANTAGES OF USING C. ELEGANS AS A MODEL ORGANISM IN APPLIED ANTI-AGING RESEARCH}

Although experiment with a mammalian model is compelling, it is time-consuming and limited by the presence of ethical concerns. C. elegans has been proved as a reasonable model organism for biological research on aging because of its advantageous features (Guarente and Kenyon, 2000). Although its anatomical structure is simple, the tissues and organs, such as muscles, nervous system, gastrointestinal tract, and gonads of C. elegans, are similar to that of higher animals (Jorgensen and Mango, 2002). In addition, its complete genome sequence is available, and about $50 \%$ of human protein-coding sequences have identifiable homologous genes in nematodes (Kim et al., 2018). Similar to humans and other higher mammals, its behavior changes and descending physiological indexes are accompanied by aging. Moreover, there are highly evolutionary conserved mechanisms controlling physiological phenomena, such as development, aging, and disease. Homologous or functionally similar forms of the main enzymes, genes, and transcription factors involved in metabolism have been found in higher animals and C. elegans (Chen et al., 2013). For example, the important transcription factor forkhead box O (FOXO), which is involved in longevity, stress resistance, and metabolism, is present in drosophila, nematodes, rodents, and humans (Martins et al., 2016). Therefore, C. elegans is broadly utilized in screening for natural bioactive substances (Ye et al., 2020). Numerous transgenes and mutants related to the longevity and aging of $C$. elegans are available (Chen et al., 2015), and many polyphenols have been successfully tested for their effects on general health benefits and longevity on nematodes.

At present, most of the bioactive substances with anti-aging activity were first discovered by using nematodes as model organisms. Since the first use of nematodes by Brenner as a tool in genetics research (Brenner, 1974), the model has been applied to many other research fields, such as development, disease modeling, metabolism, medicine, screening, and others. We also took advantages of this model organism in aging and signal transduction (Zheng et al., 2018; Qu et al., 2020b). Since two American scientists, Friedman and Johnson, discovered in the 1980s that the mutation of a single gene in nematodes can increase lifespan (Johnson and Wood, 1982; Friedman and Johnson, 1988), the genetic control of aging has rapidly developed. It is reported that aging and aging-related diseases are controlled by signaling pathways, such as autophagy-related target of rapamycin (TOR) signaling pathway (McCormick et al., 2011; Laplante and Sabatini, 2012), insulin/insulinlike growth factor 1 (IGF-1) signaling (IIS) pathway (Barbieri et al., 2003; Lapierre and Hansen, 2012), mitochondrialrelated functional signaling pathway (Sohal and Orr, 2012), and adenosine monophosphate (AMP)-activated protein kinase (AMPK) signaling pathway related to cell energy homeostasis (Salminen and Kaarniranta, 2012; Qu et al., 2020a).

\section{POLYPHENOLS}

Polyphenols are the most widely distributed group of phytochemicals (Table 1). They are usually classified into flavonoids, phenolic acids, and non-flavonoids. Flavonoids are subdivided into flavonols, flavanones, isoflavones, anthocyanins, and flavan-3-ols according to their chemical structure. Phenolic acids are subdivided into hydroxybenzoic acid and hydroxycinnamic acid. Non-flavonoids are subdivided into lignans, stilbenes, and tannins (Papaevgeniou and Chondrogianni, 2018; Fraga et al., 2019; Majidinia et al., 2019). The category is illustrated in Figure 1.

Polyphenols exert beneficial effects on health, owing to their anti-oxidant and anti-inflammatory activities, and they have been commonly employed to treat cancer, autoimmune diseases, type 2 diabetes, cardiovascular disorders, and other diseases. The structural characteristics of the carbocyclic ring of polyphenols and the number of hydroxyl groups on the ring are the main prerequisites for prolonging lifespan (Grunz et al., 2012). In this article, we reviewed literature regarding the anti-aging properties of each specific polyphenol. Different classes of chemicals might activate similar signaling pathways involved in aging processes, and one class of chemicals might be involved in multiple pathways. For example, it was reported that resveratrol can extend C. elegans lifespan through the MPK-1/ERK or SIR2.1/DAF-16 pathway (Yoon et al., 2019). In addition, many kinds of polyphenols can modulate longevity through the IIS pathway, especially through the key transcription factor DAF-16 in the pathway, for example, myricetin (Buchter et al., 2013), blueberry extract (Wang et al., 2018), echinacoside (Wang et al., 2015), and 
TABLE 1 | Modulation of the lifespan in Caenorhabditis elegans by polyphenols.

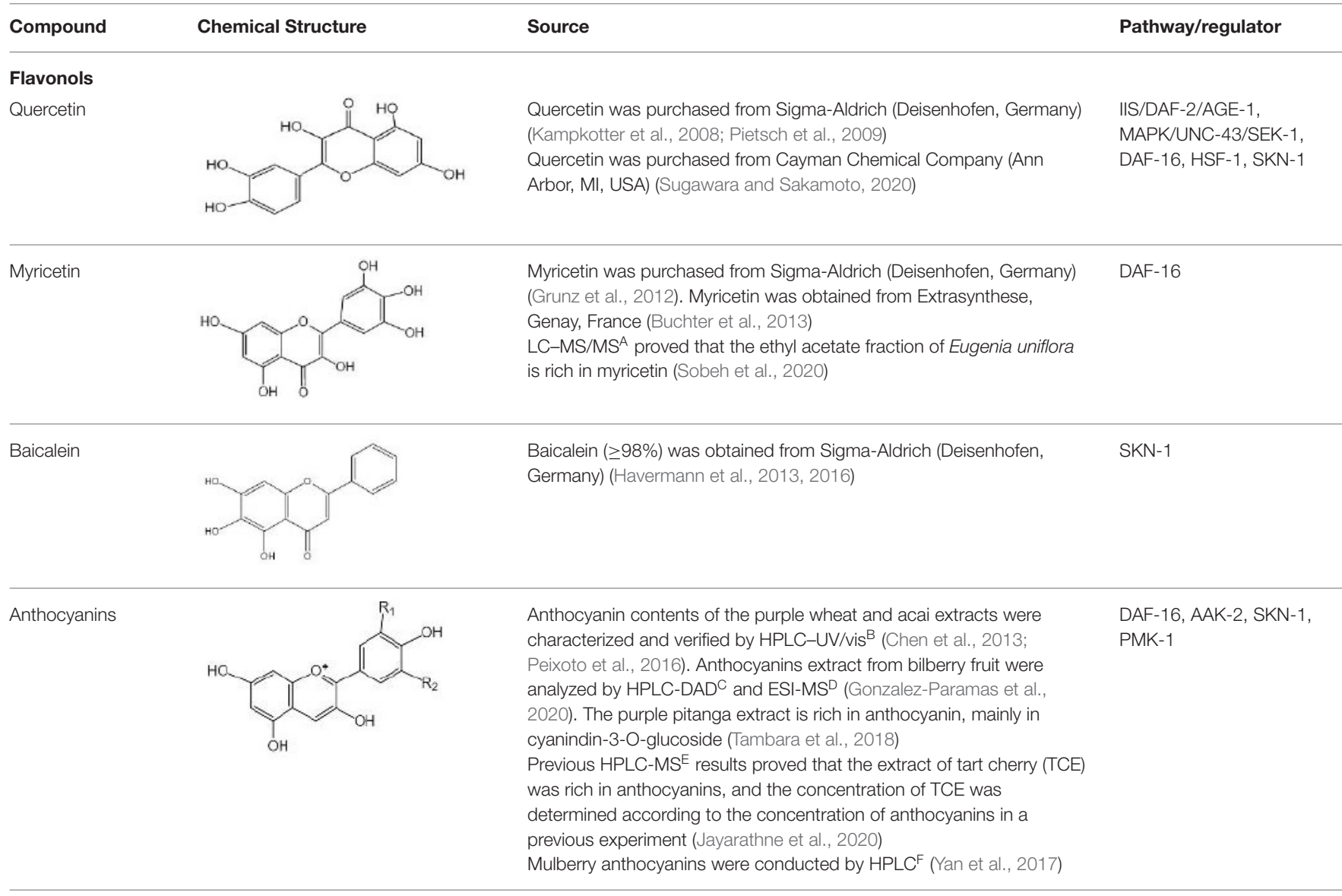

Flavan-3-ols

Catechin acid<smiles>Oc1cc(O)c2c(c1)OC(c1ccc(O)c(O)c1)[C@H](O)C2</smiles>

Epigallocatechin-3gallate (EGCG)<smiles>O=C(O)C1Cc2c(O)cc(O)cc2OC1C1CC(O)C(O)C(O)C1</smiles>

Catechin acid (96\%, HPLC) was obtained from Sigma-Aldrich (St Louis, MO, USA) (Wu et al., 2020)
BEC-1, PINK-1 
TABLE 1 | Continued

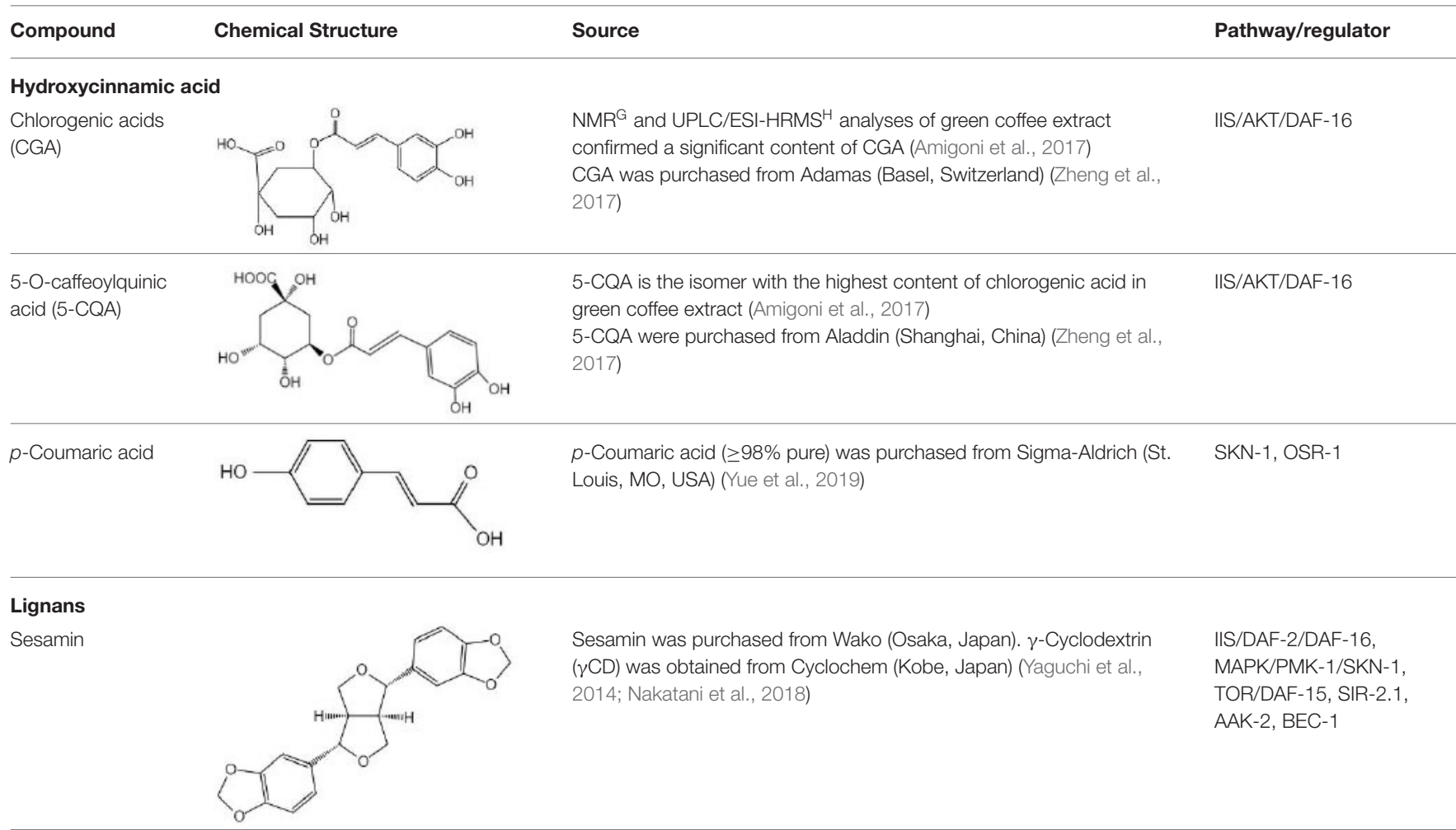

Stilbenes

Resveratrol<smiles>Oc1ccc(/C=C/c2cc(O)cc(O)c2)cc1</smiles>

Resveratrol was from purchased from Sigma-Aldrich (St. Louis, MO, USA) (Morselli et al., 2010; Lee et al., 2016; Yoon et al., 2019)
SIR-2.1, DAF-16, AAK-2, MPK-1, BEC-1

\section{Tannins}

Tannic acid

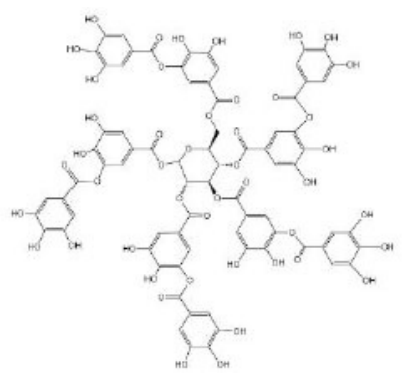

Oenothein B (OEB)

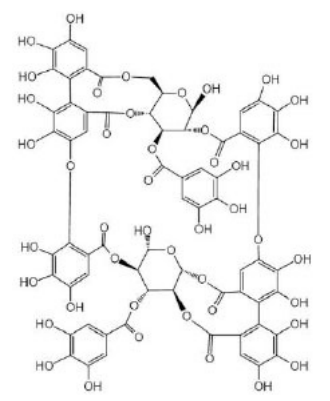

Tannic acid was purchased from Sigma-Aldrich (Taufkirchen, Germany) SEK-1, eat-2

(Saul et al., 2010, 2011) 
TABLE 1 | Continued

\begin{tabular}{|c|c|c|c|}
\hline Compound & Chemical Structure & Source & Pathway/regulator \\
\hline $\begin{array}{l}\text { Pentagalloyl } \\
\text { glucose (PGG) }\end{array}$ & & $\begin{array}{l}\text { PGG was isolated and purified from eucalyptus leaves and was } \\
\text { confirmed by HPLC and } \mathrm{MS}^{\lrcorner} \text {(Chen et al., 2014) }\end{array}$ & $\begin{array}{l}\text { IIS/AGE-1/DAF-16, SIR-2.1, } \\
\text { eat-2, isp-1 }\end{array}$ \\
\hline
\end{tabular}

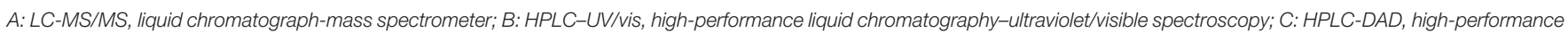

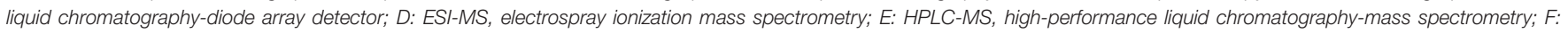

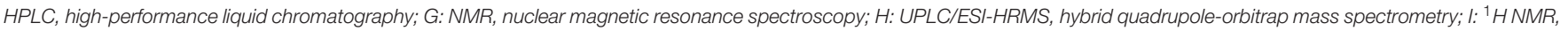
${ }^{1} \mathrm{H}$ nuclear magnetic resonance spectroscopy; J: MS, mass spectrometer.

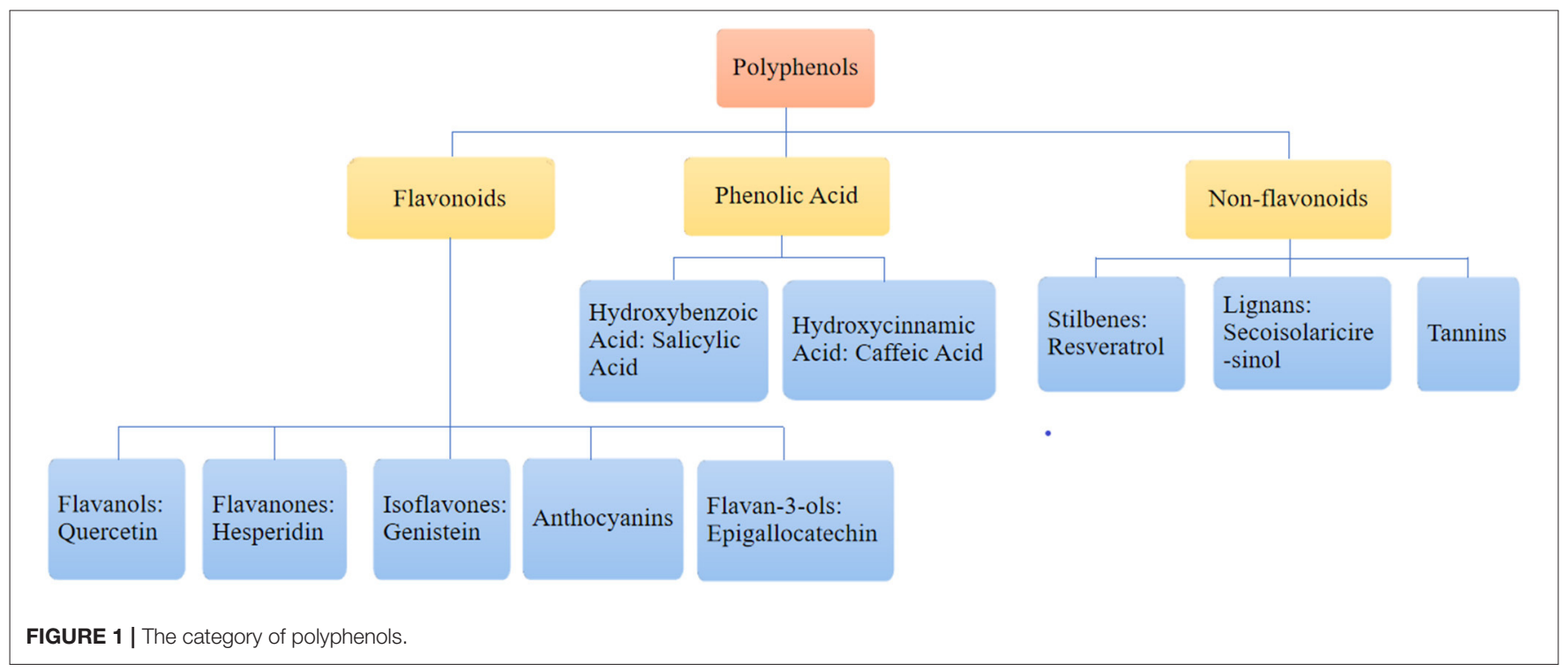

others. The main reason for this effect might be that the DAF-16 expression increases the ability to scavenge free radicals and resist oxidative stress.

\section{FLAVONOLS}

Bioactive phytochemicals, such as flavonols, are abundant in fruits and vegetables, such as onions, peppers, cauliflower, and grapes. The most common flavonol is quercetin, and other common flavonols are kaempferol, myricetin, isorhamnetin, tamarixetin, morin, fisetin, apigenin, and luteolin (Adebamowo et al., 2005; Perez-Vizcaino and Duarte, 2010). Figure 2 illustrates the model of how flavonols are involved in lifespan regulation.

Quercetin, as a strong anti-oxidant, has been demonstrated to have a positive effect on longevity and stress resistance in various animal models, and its activity and mechanism have also been studied in nematodes (Pietsch et al., 2012;
Proshkina et al., 2016). Several studies have confirmed that quercetin accumulates in nematodes and exhibits reactive oxygen species (ROS) scavenging activity, which might be the reason for its beneficial health effects, and this process is regulated by the transcription factor DAF-16 (Kampkotter et al., 2008; Sugawara and Sakamoto, 2020). The C. elegans gene, daf-16, is homologous to the mammalian gene for the FOXO transcription factor, which plays a key role in controlling several stress response signaling cascades, aging processes, and other important biological functions, and it is also considered as an important downstream factor of the IIS pathway, which is one of the main pathways that regulate the lifespan of nematodes. It starts from the DAF-2 insulin receptor, and it is also the ortholog of the insulin/IGF-1 receptor in C. elegans, through AGE-1/PI3K to AKT-1/2 and then to the downstream target DAF-16/FOXO transcription factor, to control the lifespan and metabolism of C. elegans. However, the conclusion is contrary. Some reports suggested 


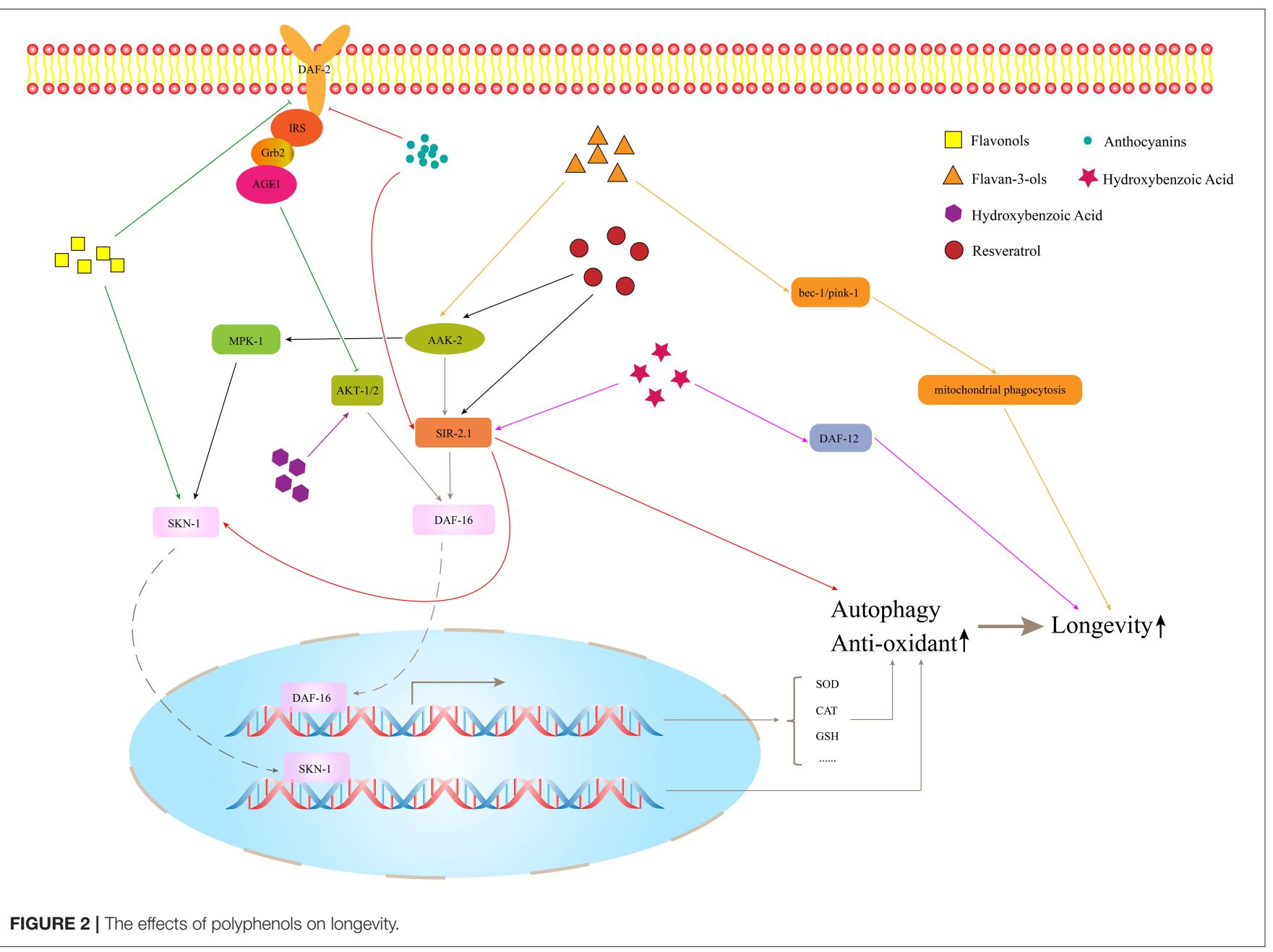

that, although DAF-2 and other components of the IIS pathway mediate the anti-oxidant activity and life-prolonging effects of quercetin on nematodes, these effects seem to be independent of DAF-16 (Pietsch et al., 2009). Hence, the role of DAF-16 in quercetin-induced health effects requires further investigation. Besides DAF-16, SKN-1 and mitogen-activated protein kinase (MAPK) pathways are also involved in the process of scavenging ROS, lifespan extension, and health improvement in C. elegans. Quercetin also induces heat resistance by coactivating the expression and/or activity of HSF-1 and DAF16 (Sugawara and Sakamoto, 2020). HSF orthologous, HSF-1, as a transcriptional regulator of stress-induced gene expression in worms, induces the expression of molecular chaperones. UNC-43 and SEK-1 seem to be parts of the lifespan regulation of quercetin (Pietsch et al., 2009). SEK-1 is an indispensable MAPK in innate immunity, and UNC-43 is a part of the SEK-1 upstream neuron regulation signal pathway. These two regulators belong to the MAPK pathway, a major immune signaling pathway (Troemel et al., 2006). UNC-43 is also a type II $\mathrm{Ca}^{2+} /$ calmodulin-dependent kinase (CAMKII) that can also regulate osmotic pressure. Therefore, quercetin can be considered a multitarget nutrient.
In addition, myricetin was first discovered by Spanier for its activation of DAF-16 and its increase in the expression of its downstream gene sod-3, but it was found that the activation of DAF-16 was not the cause of the extended lifespan because it was found that DAF-16 activation was not correlated with the myricetin-mediated decrease in mitochondrial ROS and the increase in longevity (Grunz et al., 2012). However, further experiments proved that myricetin does exert its anti-oxidant effect through DAF-16 (Buchter et al., 2013; Sobeh et al., 2020). All these studies found that, in DAF-16 mutants, the ROSscavenging effect of myricetin was blocked to a great extent, and the beneficial effect on lifespan also disappeared completely. This indicates that, although myricetin is a strong anti-oxidant, its effect on the lifespan of C. elegans is heavily dependent on DAF16 rather than its direct anti-oxidant capacity. At present, the study of myricetin on the life extension of nematodes is limited to its regulation of the IIS pathway. In the future, additional pathways should be further analyzed in this respect, and other mechanisms for myricetin-mediating health effects should be investigated (Buchter et al., 2013).

Baicalein mainly comes from Huangqin, which is one of the commonly used traditional Chinese medicines. It has been 
demonstrated that baicalein mediates anti-oxidant effects by activating nuclear factor erythroid 2-related factor 2 (Nrf2) in mammalian cell lines. When it comes to C. elegans, SKN1 is the homologous gene of the mammalian transcription factor Nrf2 (An and Blackwell, 2003). Similar to Nrf2, SKN1 can also be activated by oxidative stress or exogenous bioactive substances; then, it can be transferred to the nucleus and combined with anti-oxidant response elements (AREs) of various anti-oxidant or protective gene promoter regions. This pathway can induce a variety of anti-oxidant enzymes as the key defense mechanism against oxidative stress. The lifespan of $s k n-1$ mutant is shortened, and the resistance to oxidative stress is reduced. SKN-1 is the direct target of the IIS pathway and MAPK pathway and has some common downstream targets with DAF-16. It is also required for dietary restriction (DR)-induced longevity because it interacts with amino acid and lipid metabolism during starvation (Dall and Faergeman, 2019). It has been reported that baicalein can modulate the lifespan and stress resistance of nematodes via SKN-1, but not DAF-16, a result similar to what was obtained in mammalian cell lines (Havermann et al., 2013, 2016).

\section{ANTHOCYANINS}

Anthocyanins are found in a wide variety of colored vegetables, fruits, and cereal, especially in various berry fruits, such as bilberries, blueberries, blackberries, blackcurrants, chokeberries, strawberries, and elderberries (Chen et al., 2013; Wallace and Giusti, 2015; Yan et al., 2017). Many studies have focused on the anti-oxidant capacity of different plant extracts rich with anthocyanins. The vast majority of plant extracts rich in anthocyanins, such as extracts of purple wheat (Chen et al., 2013), acai berry (Peixoto et al., 2016), mulberry (Yan et al., 2017), purple pitanga fruit (Tambara et al., 2018), tart cherry (Jayarathne et al., 2020), and bilberry (Gonzalez-Paramas et al., 2020), which can play their beneficial role by increasing nuclear translocation of DAF-16 and promoting the expression of anti-oxidant genes, such as sod-3, and the heat shock gene, hsp-16.2, in its downstream. Heat shock proteins (HSPs) are molecular chaperones and play important roles in the protection of molecular damage under environmental stress and have the ability to maintain proteostasis and prolong the longevity of organisms (Swindell, 2009). HSP-16.2 family is expressed under stress conditions and can be considered as stress-sensitive reporters to evaluate lifespan (Strayer et al., 2003). DAF-16 is a key protein for the anti-aging effects. Mechanistically, recent studies have found that anthocyanins could regulate the AAK-2/AMPK signaling pathway to perform its biological function (Jayarathne et al., 2020). aak-2 is the encoding gene of AMPK in nematodes. AMPK is a regulator of cellular energy homeostasis, which is essential for the metabolic regulation of nematodes during starvation and diapause (Demoinet and Roy, 2018), it can be activated under low energy conditions and can maintain the steady state of energy, linking nutritional availability with longevity (Tullet, 2015). Overexpression of AAK-2 in nematodes prolongs its lifespan, and this effect also requires the downregulation of the IIS pathway and the upregulation and transposition of DAF-16 (Zhao et al., 2017). Additionally, mulberry anthocyanins can also activate transcription factors SKN-1/Nrf2 and PMK-1/MAPK and their downstream targets that are related to oxidative stress (Yan et al., 2017).

\section{FLAVAN-3-OLS}

Flavan-3-ols include catechin, gallocatechin, epicatechin, epigallocatechin, epicatechin-3-gallate, epigallocatechin-3gallate (EGCG), theaflavin, theaflavin-3-gallate, theaflavin- $3^{\prime}$ gallate, theaflavin- $3^{\prime}$-digallate, and thearubigins. Flavan-3-ols are mainly found in tea, apples, wine, and cocoa (Lei et al., 2016). Figure 2 shows the effect of flavan-3-ols on the lifespan of nematode. There are many types of flavan-3-ols, but the current research has focused mostly on tea extracts and the certain classes of flavan-3-ols, such as catechinic acid (CA) and EGCG. Xiong et al. (2014) found that the black tea extracts contain a variety of flavan-3-ols, which can increase the lifespan of C. elegans under stress conditions, such as osmotic pressure imbalance, ultraviolet radiation, and heat stress. This effect might be mediated by the SEK-1 signaling and SIR-2.1/DAF-16/SOD-3 pathways, which could increase stress resistance. Worms simultaneously treated with catechins-rich green tea aqueous extract (GTE) and a lethal dose of pro-oxidant, i.e., juglone, showed a decreased expression of $h s p-16.2$ and a significantly increased survival rate as compared with worms not receiving GTE. It suggested that GTE could enhance the anti-stress ability of nematodes and reduce oxidative damage in vivo (Abbas and Wink, 2014). In addition, it was found that $\mathrm{CA}$, as a natural polyphenol compound, extended the lifespan and declined the age-related behaviors of $C$. elegans by regulating the mitophagy pathway related to genes of bec-1 and pink-1. It was found that it can act in the role of inducing mitochondrial phagocytosis at the early stage, which was also a key period to affect the lifespan (Wu et al., 2020). Mitophagy can prevent the accumulation of dysfunctional mitochondria and prolong lifespan. EGCG is another widely studied flavane. The regulation of EGCG on the lifespan of nematodes is affected by the concentration. The effect on the organism can be described as a hormetic effect; in other words, stimulatory and inhibitory effects would be generated in low and high doses. The health effects of EGCG depend on the hormetic effect. It was found that, when the concentration was $<25 \mu \mathrm{M}$, it could prolong the lifespan of nematodes under the stress and improve their stress ability and the partial decline of age-related physiological behavior, but it was not enough to affect the lifespan of worms under normal conditions (Brown et al., 2006; Zhang et al., 2009). When the concentration is above $800 \mu \mathrm{M}$, it might produce toxic effects (Xiong et al., 2018). At a suitable concentration, EGCG induced ROS in a time-resolved manner, which can temporarily increase ROS level in the early stage and activate AAK-2/AMPK, change the metabolism of $\mathrm{NAD}^{+}$, and then increase the expression of its downstream target protein SIR-2.1. Previous studies have found that EGCG can increase the nuclear translocation and expression of DAF-16 
and activate its downstream antioxidant genes (Zhang et al., 2009; Bartholome et al., 2010). However, the upstream regulation mechanism has not been further studied. Currently, it was found that EGCG acted on SIR-2.1 instead of the IIS pathway to regulate DAF-16 (Xiong et al., 2018). Besides, EGCG can mainly restore the mitochondrial function and increase the biogenesis of early-to-mid adult worms, thus improving the redox steady state of worms. The EGCG-induced longevity of nematodes also depends on the mitochondrial function. The health effect would decrease gradually with age increases (Xiong et al., 2018). Sirtuin of C. elegans is the closest homolog to human SIRT1, which is encoded by the gene sir-2.1. It is also a conservative transcription regulator. As an $\mathrm{NAD}^{+}$-dependent histone deacetylase, the overexpression of sirtuin can prolong the lifespan of many species (Smith et al., 2014; Seo et al., 2015). Sirtuin can directly activate DAF-16/FOXO by deacetylation, which affects the lifespan independently of IIS (Kenyon, 2010). It can also induce autophagy by upregulating the autophagy gene and inhibiting the TOR signal together with AMPK (Ruderman et al., 2010). In addition, the anti-oxidant mechanism is activating SKN-1 and regulating lifespan through the pathway, partially overlapping with DR (Jung et al., 2017). DR is one of the most influential environmental interventions for prolonging the lifespan and healthspan of many species.

\section{HYDROXYBENZOIC ACID}

Hydroxybenzoic acid is widely distributed in vegetables and fruits and can be synthesized from polyphenols by gut bacteria. It has been confirmed to activate Nrf2 (Juurlink et al., 2014), suggesting that it might have the anti-aging effects on nematodes via the Nrf2 signaling pathway. Furthermore, 4hydroxybenzoic acid could extend the lifespan of nematodes through the activation of DAF-16/FOXO mediated by SIR2.1/SIR-2, which demonstrated no relationship with DR and IIS pathway. It can also increase the stress resistance under osmotic, heat, and oxidative stress conditions (Kim et al., 2014). By bioinformatics analysis, aspirin was found to alter the expression of genes, which are involved in fat metabolisms, such as acs-2, ech-1.2, and cpt-5, which can extend the longevity of C. elegans through the activation of DAF-12 and DAF-16 (Huang et al., 2017). DAF-12 is a nuclear hormone receptor that can be initiated by insulin/IGF-1 and TGF- $\beta$ and plays an important role in metabolism, longevity, and reproductive development in C. elegans. As is known to all, the primary component of aspirin is salicylic acid. As an isomer of hydroxybenzoic acid, it suggests that hydroxybenzoic acid might influence the expression of genes involved in anti-oxidation and fat metabolism.

\section{HYDROXYCINNAMIC ACID}

Hydroxycinnamic acid and its derivative, caffeic acid, are abundant in tea leaves, red wine, and coffee. It was reported that extracts of green coffee beans (GCEs), which are composed mainly of chlorogenic acid (CGA) and its derivative, 5caffeoylquinic acid (5-CQA), have beneficial effects on longevity and reproduction in $C$. elegans. The study also indicated that, compared with CGEs rich in pure 5-CQA, the CGEs rich in 5-CQA have a stronger anti-aging effect, which strongly supports that it might be a better choice to use the mixture of bioactive compounds instead of just one single bioactive molecule (Amigoni et al., 2017). At the same time, it was also found that CGA and its isomers, such as 5-CQA and 4caffeoylquinic acid (4-CQA), acted on the upstream of AKT in the IIS pathway and then exerted their life-prolonging and anti-aging effects mainly via DAF-16 and its downstream stress factors, HSF-1, SKN-1, and HIF-1 (Zheng et al., 2017). Moreover, p-coumaric acid, another derivative of hydroxycinnamic acid, can enhance the ability to resist SKN-1-mediated oxidative stress and OSR-1-mediated osmotic stress (OSR-1 can negatively regulate the activity of the MAPK pathway) (Yue et al., 2019).

\section{LIGNANS}

Six lignans were isolated from Arctium lappa seeds, and all of them were found to have anti-aging properties and to upregulate the expression of daf-16 and jnk-1 (Su and Wink, 2015). jnk-1 is considered as a positive regulator of $d a f-16$, which indicates that lignans have the life-promoting activity through the JNK-1/DAF16 cascade. Sesamin is a major lignan constituent of sesame and possesses various health-promoting effects. That sesamin could not only prolong the life of nematodes but could also reduce the toxicity of Alzheimer's disease (AD) $\beta$-amyloid $(A \beta)$ plaque (Keowkase et al., 2018). It was also found that the resistance of nematodes to physical stress and some pathogenic bacteria could not be enhanced by sesamin, but it could protect nematodes from oxidative stress caused by toxins, which is partly due to the indirect hormetic effect of sesamin. Besides, it was found that sesamin could play the anti-aging role via the genes constituting the IIS pathway (daf-2 and daf-16) and MAPK pathway (pmk1 and skn-1) (Yaguchi et al., 2014). PMK-1 is a kinase that plays an important role in immune defense and longevity in the MAPK pathway. In addition, sesamin can also act as a mimic of DR. Sesamin depends on SIR-2.1/SIRT1, AAK-2/AMPK, an autophagic modulator BEC-1, and daf-15, which encodes the target of TOR-binding partner raptor, to promote longevity (Yaguchi et al., 2014; Nakatani et al., 2018). The inhibition of the TOR pathway is another well-known intervention method to prolong lifespan. DR might induce autophagy and activate DAF-16 by inhibiting TOR kinase (Cypser et al., 2013). BEC-1 is necessary for the longevity induced by overexpression of sir2.1. SIRT1, TOR, and AMPK are currently known as signaling pathways associated with DR. Unlike other DR analogs, sesamin is likely to be involved in almost all of the known DR-related pathways, which can prolong lifespan.

Another lignan, i.e., pinoresinol has been observed to increase the nuclear translocation of DAF-16, but it has no effect on the longevity of nematodes and has no regulating ability to the stress resistance and oxidation resistance. Although it shows strong 
oxidation resistance in vitro, its functional effects in organisms need further study at a molecular level (Koch et al., 2015).

\section{STILBENES}

The most important representative of stilbene compounds is resveratrol, which is mainly derived from grape skins, grape seeds, red wine (Salehi et al., 2018), blueberries, peanuts, and some traditional Chinese herbal medicines, such as rhubarb (Malaguarnera, 2019), and polygonum cuspidatum (Zhang, 2006). Resveratrol is usually recommended as a dietary supplement to maintain redox balance and to delay aging (Desjardins et al., 2017).

The activation of sirtuins is considered to be an important mechanism of resveratrol-mediated longevity. Research found that resveratrol can activate SIR-2.1 and then prolong the life of nematodes by regulating bec-1 to induce autophagy (Morselli et al., 2010). Lee et al. (2016) found that resveratrol does not need to exert its health effects through DAF-16 after activating SIR-2.1, which indicates that there might be other regulatory pathways downstream of SIR-2.1, whereas Yoon et al. (2019) found that SIR-2.1 relies on DAF-16 for its function, so the role of DAF-16 in resveratrol-induced longevity needs further study. As research progresses, scientists have learned more about the mechanism of how resveratrol works to delay aging. The effect of resveratrol on life extension might not work entirely in a sirtuin-dependent way. As a DR analog, resveratrol can prolong lifespan through AAK-2, a key factor in the AMPK pathway, and without the association of DAF-16. Similar to SIR-2.1, MPK-1 is also one of the key regulators for lifespan extension (Yoon et al., 2019). However, its contribution to resveratrol-mediated life extension is completely independent of SIR-2.1, and they have different downstream regulatory genes. MPK-1 is also known as human ERK homo that acts by promoting downstream SKN-1 nuclear translocation and is first identified as a longevity factor (Okuyama et al., 2010). Resveratrol can alleviate the damage caused by ROS and prolong the life of nematodes under pressure (Chen et al., 2013). In addition, the two newly synthesized resveratrol derivatives have stronger biological and anti-oxidant activity than resveratrol. Their strong anti-oxidant ability can also regulate DAF-16, SKN1, SIR-2.1 in the redox activity signal pathway (Fischer et al., 2017).

\section{TANNINS}

Tannic acid (TA) belongs to the hydrolyzable tannins, containing five digallic acid residues covalently linked to a central glucose molecule, and it can precipitate protein. As a strong anti-oxidant, the observed increase in heat stress resistance and oxidative stress resistance is not due to its ability to directly remove oxygen free radicals but its ability to act as a stimulus to activate the anti-oxidant system of the body (Saul et al., 2010, 2011). Studies have shown that a low concentration of TA might simulate mild pathogenic stress, strengthen the SEK-1-based pathogen defense system, inhibit the potentially harmful effects of TA, and induce nematodes to prolong their life effectively (Saul et al., 2010). In addition, TA itself does not reduce the food intake of nematodes, but exerts the molecular regulation through eat-2 in the DR pathway or precipitates and combines nutritional proteins and digestive enzymes (eat-2 mutant suffered from insufficient food intake due to decreased pharyngeal pumping). It is worth noting that the concentration range of health effects of TA is relatively narrow, so it is also very important to find a suitable concentration to treat C. elegans. Unlike TA, ellagic acid (EA) can be used as a chemical repellent to reduce the feeding behavior of nematodes and prolong the lifespan of nematodes by its strong antibacterial ability (Saul et al., 2011). Oenothein B (Chen et al., 2020) and pentagalloyl glucose (Chen et al., 2014) extracted from eucalyptus leaves can prolong healthy life by regulating multiple targets. They can regulate the IIS pathway via age-1 and daf16 , the DR pathway via eat-2 and sir-2.1, and mitochondrial electron transfer chain via isp-1 to promote healthy life, including reducing age pigment and ROS accumulation and improving exercise flexibility, heat stress tolerance, and lifespan. isp- 1 is one of the genes encoding mitochondrial electron transport chain components, and the deletion of isp- 1 exists in the respiratory chain complex III. Their mechanism of action might be the same because of their similar structure.

\section{CONCLUSIONS}

Before studying the signaling pathway implicated in a certain disease in a model organism, some researchers first test this pathway in suitable cell lines. For example, resveratrol was found in the generation of different effects, such as promoting proliferation in mesenchymal stem cells, with possible involvement of the ERK/GSK-3 $\beta$ pathway (Yoon et al., 2015). Nematodes were further utilized to test the MPK-1 (an ERK homolog) signaling (Yoon et al., 2019). When we treat nematodes with plant extracts, we can first analyze each extract component using mass spectrometry, high-performance liquid chromatography, or similar methods; this analysis might help to identify the key components responsible for the biological effects.

At present, researchers rarely treat $C$. elegans with polyphenols, such as flavanones and isoflavones; therefore, it is unknown whether or not these polyphenols have direct effects on C. elegans. However, many studies demonstrated that the abovementioned polyphenols can act on homologous genes of C. elegans in other species and that these genes are involved in aging and regulation of lifespan. Flavanones, of which the representative molecule is hesperidin, are mainly found in citrus plants and have been demonstrated to be able to reduce oxidative stress caused by a high-fat diet in mice and to slow down the aging process in old-aged rats. Some studies found that one of the targets of flavanones in animals is Nrf2, whereas C. elegans has an Nrf2-homolog gene, SKN-1 (Ferreira et al., 2016; Barreca et al., 2017; Habtemariam, 2019; Miler et al., 2020). Isoflavones, such as genistein and daidzein, are generally regarded as phytoestrogens; there is evidence that Nrf2 is also one of the downstream targets of isoflavones and that it can 
also regulate fat metabolism in rats with diet-induced obesity, acting through the AMPK pathway (Li and Zhang, 2017; Krizova et al., 2019). In conclusion, further studies should be conducted to test whether these polyphenols have beneficial effects on C. elegans.

\section{PROSPECTS}

To the best of our knowledge, in the study of total polyphenols in plants, especially medicinal plants, their functions, the bioactive substances, and molecular mechanisms for prolonging lifespan and delaying senescence have not received enough attention. For example, mulberry leaves have been widely used in traditional Chinese medicine and folk dietary therapy for their outstanding effects of detoxifying the liver, improving eyesight, and prolonging life. It is believed that mulberry leaf extracts used in traditional Chinese medicine have anti-oxidant and hepatoprotective effects, and those two activities are related to mitochondria function (Meng et al., 2020). Liver tissue contains a large number of mitochondria, and the fatty acids are activated into ester-acyl-coenzyme $\mathrm{A}$, which is metabolized by $\beta$ oxidation in mitochondria. Acetyl-coenzyme A and fat synthetases required for fatty acid synthesis come from mitochondria. At present, mulberry leaf extract has been confirmed to have beneficial effects on several diseases, such as cancer, type 2 diabetes, and obesity. In addition, modern medicine experiments have proved that mulberry leaf extract can delay aging in mice (Lim et al., 2013; Turgut et al., 2016). Mulberry leaf extract is an effective and natural free-radical scavenger and anti-oxidant, but the research on mulberry leaf extract and mulberry leaf polyphenol is limited to its anti-oxidant activity in vitro, and its specific mechanisms of action have not been elaborated. Moreover, the activities of mulberry leaf polyphenols have not yet been associated with any specific physiological functions. Besides, nematodes being treated by the combination of two extracts from different plants

\section{REFERENCES}

Abbas, S., and Wink, M. (2014). Green tea extract induces the resistance of caenorhabditis elegans against oxidative stress. Antioxidants 3, 129-143. doi: 10.3390/antiox3010129

Adachi, H., and Ishii, N. (2000). Effects of tocotrienols on life span and protein carbonylation in Caenorhabditis elegans. J. Gerontol. A Biol. Sci. Med. Sci. 55, B280-285. doi: 10.1093/gerona/55.6.B280

Adebamowo, C. A., Cho, E., Sampson, L., Katan, M. B., Spiegelman, D., Willett, W. C., et al. (2005). Dietary flavonols and flavonol-rich foods intake and the risk of breast cancer. Int. J. Cancer 114, 628-633. doi: 10.1002/ijc. 20741

Amigoni, L., Stuknyte, M., Ciaramelli, C., Magoni, C., Bruni, I., De Noni, I., et al. (2017). Green coffee extract enhances oxidative stress resistance and delays aging in Caenorhabditis elegans. J. Funct. Foods 33, 297-306. doi: 10.1016/j.jff.2017.03.056

Amor, C., Feucht, J., Leibold, J., Ho, Y. J., Zhu, C., Alonso-Curbelo, D., et al. (2020). Senolytic CAR T cells reverse senescence-associated pathologies. Nature 583, 127-132. doi: 10.1038/s41586-020-2403-9

An, J. H., and Blackwell, T. K. (2003). SKN-1 links C. elegans mesendodermal specification to a conserved oxidative stress response. Genes Dev. 17, 1882-1893. doi: 10.1101/gad.1107803 revealed stronger effects than the treatment with only either of the single extract. A recent study shows that nematodes treated with the mixtures of blueberry and apple peel extracts have a longer lifespan than those treated with only one substance (Song et al., 2020a,b). Could mulberry leaf extract exert the effects observed in traditional Chinese medicine by regulating fat metabolism? What are the specific anti-aging mechanisms of mulberry leaves in C. elegans? Could the combinations of mulberry leaves, polyphenols, and other polyphenols, or other bioactive substances play their beneficial roles more significantly, and what are their mechanisms? We would like to answer these questions by conducting further research.

\section{AUTHOR CONTRIBUTIONS}

ZQ and NL conceived the idea and wrote the manuscript with input from LL, PG, and SZ. PG and PW prepared the figures. LL, PW, and SZ prepared the tables. All authors edited and approved the final manuscript.

\section{FUNDING}

This review was supported by the National Natural Science Foundation of China (Nos. 81872584 and 81472941), the National 863 Young Scientist Program (No. 2015AA020940), the Natural Science Foundation of Guangdong Province (No. 2016A030313138), Key R \& D and Promotion Project of Henan Province (No. 192102310259), Key Scientific Research Project of Henan Province (No. 21A330001), the Key Projects of Guangzhou Science and Technology Program (No. 201704020056), Interdisciplinary Research for First-Class Discipline Construction Project of Henan University (No. 2019YLXKJC04), the Scientific Research Project for University of Education Bureau of Guangzhou (No. 201831841), and the Yellow River Scholar Foundation of Henan University.

Barbieri, M., Bonafe, M., Franceschi, C., and Paolisso, G. (2003). Insulin/IGFI-signaling pathway: an evolutionarily conserved mechanism of longevity from yeast to humans. Am. J. Physiol. Endocrinol. Metab. 285, E1064-E1071. doi: 10.1152/ajpendo.00296.2003

Barreca, D., Gattuso, G., Bellocco, E., Calderaro, A., Trombetta, D., Smeriglio, A., et al. (2017). Flavanones: Citrus phytochemical with health-promoting properties. Biofactors 43, 495-506. doi: 10.1002/biof.1363

Bartholome, A., Kampkotter, A., Tanner, S., Sies, H., and Klotz, L. O. (2010). Epigallocatechin gallate-induced modulation of FoxO signaling in mammalian cells and C. elegans: FoxO stimulation is masked via PI3K/Akt activation by hydrogen peroxide formed in cell culture. Arch. Biochem. Biophys. 501, 58-64. doi: $10.1016 /$ j.abb.2010.05.024

Brenner, S. (1974). The genetics of Caenorhabditis elegans. Genetics 77, 71-94. doi: 10.1093/genetics/77.1.71

Brown, M. K., Evans, J. L., and Luo, Y. (2006). Beneficial effects of natural antioxidants EGCG and alpha-lipoic acid on life span and age-dependent behavioral declines in Caenorhabditis elegans. Pharmacol. Biochem. Behav. 85, 620-628. doi: 10.1016/j.pbb.2006.10.017

Buchter, C., Ackermann, D., Havermann, S., Honnen, S., Chovolou, Y., Fritz, G., et al. (2013). Myricetin-mediated lifespan extension in Caenorhabditis elegans is modulated by DAF-16. Int. J. Mol. Sci. 14, 11895-11914. doi: 10.3390/ijms140611895 
Chen, W., Muller, D., Richling, E., and Wink, M. (2013). Anthocyanin-rich purple wheat prolongs the life span of Caenorhabditis elegans probably by activating the DAF-16/FOXO transcription factor. J. Agric. Food Chem. 61, 3047-3053. doi: 10.1021/jf3054643

Chen, X., Barclay, J. W., Burgoyne, R. D., and Morgan, A. (2015). Using C. elegans to discover therapeutic compounds for ageing-associated neurodegenerative diseases. Chem. Cent. J. 9:65. doi: 10.1186/s13065-015-0143-y

Chen, Y., Onken, B., Chen, H., Xiao, S., Liu, X., Driscoll, M., et al. (2014). Mechanism of longevity extension of Caenorhabditis elegans induced by pentagalloyl glucose isolated from eucalyptus leaves. J. Agric. Food Chem. 62, 3422-3431. doi: $10.1021 / \mathrm{j} 5500210 \mathrm{p}$

Chen, Y., Onken, B., Chen, H., Zhang, X., Driscoll, M., Cao, Y., et al. (2020). Healthy lifespan extension mediated by oenothein B isolated from Eucalyptus grandis x Eucalyptus urophylla GL9 in Caenorhabditis elegans. Food Funct. 11, 2439-2450. doi: 10.1039/C9FO02472G

Childs, B. G., Baker, D. J., Wijshake, T., Conover, C. A., Campisi, J., and van Deursen, J. M. (2016). Senescent intimal foam cells are deleterious at all stages of atherosclerosis. Science 354, 472-477. doi: 10.1126/science.aaf6659

Childs, B. G., Durik, M., Baker, D. J., and van Deursen, J. M. (2015). Cellular senescence in aging and age-related disease: from mechanisms to therapy. Nat. Med. 21, 1424-1435. doi: 10.1038/nm.4000

Cypser, J. R., Kitzenberg, D., and Park, S. K. (2013). Dietary restriction in C. elegans: recent advances. Exp. Gerontol. 48, 1014-1017. doi: 10.1016/j.exger.2013.02.018

Dall, K. B., and Faergeman, N. J. (2019). Metabolic regulation of lifespan from a C. elegans perspective. Genes Nutr. 14:25. doi: 10.1186/s12263-019-0650-x

De la Fuente, M., Sanchez, C., Vallejo, C., Diaz-Del Cerro, E., Arnalich, F., and Hernanz, A. (2020). Vitamin C and vitamin C plus E improve the immune function in the elderly. Exp. Gerontol. 142:111118. doi: 10.1016/j.exger.2020.111118

Demoinet, E., and Roy, R. (2018). Surviving starvation: AMPK protects germ cell integrity by targeting multiple epigenetic effectors. Bioessays 40:1700095. doi: 10.1002/bies.201700095

Desjardins, D., Cacho-Valadez, B., Liu, J. L., Wang, Y., Yee, C., Bernard, K., et al. (2017). Antioxidants reveal an inverted U-shaped dose-response relationship between reactive oxygen species levels and the rate of aging in Caenorhabditis elegans. Aging Cell 16, 104-112. doi: 10.1111/acel.12528

Ferreira, P. S., Spolidorio, L. C., Manthey, J. A., and Cesar, T. B. (2016). Citrus flavanones prevent systemic inflammation and ameliorate oxidative stress in C57BL/6J mice fed high-fat diet. Food Funct. 7, 2675-2681. doi: $10.1039 / \mathrm{c} 5$ fo $01541 \mathrm{c}$

Fischer, N., Buchter, C., Koch, K., Albert, S., Csuk, R., and Watjen, W. (2017). The resveratrol derivatives trans-3,5-dimethoxy-4-fluoro-4'hydroxystilbene and trans-2,4,5-trihydroxystilbene decrease oxidative stress and prolong lifespan in Caenorhabditis elegans. J. Pharm. Pharmacol. 69, 73-81. doi: $10.1111 /$ jphp. 12657

Fraga, C. G., Croft, K. D., Kennedy, D. O., and Tomas-Barberan, F. A. (2019). The effects of polyphenols and other bioactives on human health. Food Funct. 10, 514-528. doi: 10.1039/C8FO01997E

Friedman, D. B., and Johnson, T. E. (1988). A mutation in the age-1 gene in Caenorhabditis elegans lengthens life and reduces hermaphrodite fertility. Genetics 118, 75-86. doi: 10.1093/genetics/118.1.75

Gonzalez-Paramas, A. M., Brighenti, V., Bertoni, L., Marcelloni, L., Ayuda-Duran, B., Gonzalez-Manzano, S., et al. (2020). Assessment of the in vivo antioxidant activity of an anthocyanin-rich bilberry extract using the Caenorhabditis elegans model. Antioxidants 9:509. doi: 10.3390/antiox9060509

Grunz, G., Haas, K., Soukup, S., Klingenspor, M., Kulling, S. E., Daniel, H., et al. (2012). Structural features and bioavailability of four flavonoids and their implications for lifespan-extending and antioxidant actions in C. elegans. Mech. Ageing Dev. 133, 1-10. doi: 10.1016/j.mad.2011. 11.005

Guarente, L., and Kenyon, C. (2000). Genetic pathways that regulate ageing in model organisms. Nature 408, 255-262. doi: 10.1038/35041700

Habtemariam, S. (2019). The Nrf2/HO-1 axis as targets for flavanones: neuroprotection by pinocembrin, naringenin, and eriodictyol. Oxid. Med. Cell. Longev. 2019:4724920. doi: 10.1155/2019/4724920

Havermann, S., Humpf, H. U., and Watjen, W. (2016). Baicalein modulates stressresistance and life span in C. elegans via SKN-1 but not DAF-16. Fitoterapia 113, 123-127. doi: 10.1016/j.fitote.2016.06.018
Havermann, S., Rohrig, R., Chovolou, Y., Humpf, H. U., and Watjen, W. (2013). Molecular effects of baicalein in Hct116 cells and Caenorhabditis elegans: activation of the Nrf2 signaling pathway and prolongation of lifespan. J. Agric. Food Chem. 61, 2158-2164. doi: 10.1021/jf304553g

Huang, X. B., Mu, X. H., Wan, Q. L., He, X. M., Wu, G. S., and Luo, H. R. (2017). Aspirin increases metabolism through germline signalling to extend the lifespan of Caenorhabditis elegans. PLoS ONE 12:e0184027. doi: 10.1371/journal.pone.0184027

Jayarathne, S., Ramalingam, L., Edwards, H., Vanapalli, S. A., and MoustaidMoussa, N. (2020). Tart cherry increases lifespan in Caenorhabditis elegans by altering metabolic signaling pathways. Nutrients 12:1482. doi: $10.3390 /$ nu12051482

Johnson, S. C., Rabinovitch, P. S., and Kaeberlein, M. (2013). mTOR is a key modulator of ageing and age-related disease. Nature 493, 338-345. doi: $10.1038 /$ nature 11861

Johnson, T. E., and Wood, W. B. (1982). Genetic analysis of life-span in Caenorhabditis elegans. Proc. Natl. Acad. Sci. U.S.A. 79, 6603-6607. doi: $10.1073 /$ pnas.79.21.6603

Jorgensen, E. M., and Mango, S. E. (2002). The art and design of genetic screens: Caenorhabditis elegans. Nat. Rev. Genet. 3, 356-369. doi: 10.1038/nrg794

Jung, H. Y., Lee, D., Ryu, H. G., Choi, B. H., Go, Y., Lee, N., et al. (2017). Myricetin improves endurance capacity and mitochondrial density by activating SIRT1 and PGC-1alpha. Sci. Rep. 7:6237. doi: 10.1038/s41598-017-05303-2

Juurlink, B. H., Azouz, H. J., Aldalati, A. M., AlTinawi, B. M., and Ganguly, P. (2014). Hydroxybenzoic acid isomers and the cardiovascular system. Nutr. J. 13:63. doi: 10.1186/1475-2891-13-63

Kampkotter, A., Timpel, C., Zurawski, R. F., Ruhl, S., Chovolou, Y., Proksch, P., et al. (2008). Increase of stress resistance and lifespan of Caenorhabditis elegans by quercetin. Comp. Biochem. Physiol. B Biochem. Mol. Biol. 149, 314-323. doi: $10.1016 /$ j.cbpb.2007.10.004

Kenyon, C. (2010). A pathway that links reproductive status to lifespan in Caenorhabditis elegans. Ann. N. Y. Acad. Sci. 1204, 156-162. doi: 10.1111/j.1749-6632.2010.05640.x

Keowkase, R., Shoomarom, N., Bunargin, W., Sitthithaworn, W., and Weerapreeyakul, N. (2018). Sesamin and sesamolin reduce amyloid-beta toxicity in a transgenic Caenorhabditis elegans. Biomed. Pharmacother. 107, 656-664. doi: 10.1016/j.biopha.2018.08.037

Kim, D. K., Jeon, H., and Cha, D. S. (2014). 4-Hydroxybenzoic acid-mediated lifespan extension in Caenorhabditis elegans. J. Funct. Foods 7, 630-640. doi: 10.1016/j.jff.2013.12.022

Kim, W., Underwood, R. S., Greenwald, I., and Shaye, D. D. (2018). OrthoList 2: a new comparative genomic analysis of human and Caenorhabditis elegans genes. Genetics 210, 445-461. doi: 10.1534/genetics.118.301307

Koch, K., Buchter, C., Havermann, S., and Watjen, W. (2015). The lignan pinoresinol induces nuclear translocation of DAF-16 in Caenorhabditis elegans but has no effect on life span. Phytother. Res. 29, 894-901. doi: 10.1002/ptr.5330

Krizova, L., Dadakova, K., Kasparovska, J., and Kasparovsky, T. (2019). Isoflavones. Molecules 24:1076. doi: 10.3390/molecules24061076

Lapierre, L. R., and Hansen, M. (2012). Lessons from C. elegans: signaling pathways for longevity. Trends Endocrinol Metab. 23, 637-644. doi: $10.1016 /$ j.tem.2012.07.007

Laplante, M., and Sabatini, D. M. (2012). mTOR signaling in growth control and disease. Cell 149, 274-293. doi: 10.1016/j.cell.2012.03.017

Lee, J., Kwon, G., Park, J., Kim, J. K., and Lim, Y. H. (2016). Brief communication: SIR-2.1-dependent lifespan extension of Caenorhabditis elegans by oxyresveratrol and resveratrol. Exp. Biol. Med. 241, 1757-1763. doi: $10.1177 / 1535370216650054$

Lei, L., Yang, Y., He, H., Chen, E., Du, L., Dong, J., et al. (2016). Flavan-3ols consumption and cancer risk: a meta-analysis of epidemiologic studies. Oncotarget 7, 73573-73592. doi: 10.18632/oncotarget.12017

Li, J., Bonkowski, M. S., Moniot, S., Zhang, D., Hubbard, B. P., Ling, A. J. Y., et al. (2017). AGING A conserved $\mathrm{NAD}(+)$ binding pocket that regulates protein-protein interactions during aging. Science 355, 1312-1317. doi: $10.1126 /$ science.aad8242

Li, Y., and Zhang, H. (2017). Soybean isoflavones ameliorate ischemic cardiomyopathy by activating Nrf2-mediated antioxidant responses. Food Funct. 8, 2935-2944. doi: 10.1039/C7FO $00342 \mathrm{~K}$ 
Lim, H. H., Yang, S. J., Kim, Y., Lee, M., and Lim, Y. (2013). Combined treatment of mulberry leaf and fruit extract ameliorates obesity-related inflammation and oxidative stress in high fat diet-induced obese mice. J. Med. Food 16, 673-680. doi: 10.1089/jmf.2012.2582

Majidinia, M., Bishayee, A., and Yousefi, B. (2019). Polyphenols: major regulators of key components of DNA damage response in cancer. DNA Repair 82:102679. doi: 10.1016/j.dnarep.2019.102679

Malaguarnera, L. (2019). Influence of resveratrol on the immune response. Nutrients 11:946. doi: 10.3390/nu11050946

Martins, R., Lithgow, G. J., and Link, W. (2016). Long live FOXO: unraveling the role of FOXO proteins in aging and longevity. Aging Cell 15, 196-207. doi: 10.1111/acel.12427

McCormick, M. A., Tsai, S. Y., and Kennedy, B. K. (2011). TOR and ageing: a complex pathway for a complex process. Philos. Trans. R. Soc. Lond. B Biol. Sci. 366, 17-27. doi: 10.1098/rstb.2010.0198

Meng, Q., Qi, X., Fu, Y., Chen, Q., Cheng, P., Yu, X., et al. (2020). Flavonoids extracted from mulberry (Morus alba L.) leaf improve skeletal muscle mitochondrial function by activating AMPK in type 2 diabetes. $J$. Ethnopharmacol. 248:112326. doi: 10.1016/j.jep.2019.112326

Miler, M., Zivanovic, J., Ajdzanovic, V., Milenkovic, D., Jaric, I., Sosic-Jurjevic, B., et al. (2020). Citrus flavanones upregulate thyrotroph sirt1 and differently affect thyroid Nrf2 expressions in old-aged wistar rats. J. Agric. Food Chem. 68, 8242-8254. doi: 10.1021/acs.jafc.0c03079

Morselli, E., Maiuri, M. C., Markaki, M., Megalou, E., Pasparaki, A., Palikaras, K., et al. (2010). Caloric restriction and resveratrol promote longevity through the Sirtuin-1-dependent induction of autophagy. Cell Death Dis. 1:e10. doi: $10.1038 /$ cddis.2009.8

Nakatani, Y., Yaguchi, Y., Komura, T., Nakadai, M., Terao, K., Kage-Nakadai, E., et al. (2018). Sesamin extends lifespan through pathways related to dietary restriction in Caenorhabditis elegans. Eur. J. Nutr. 57, 1137-1146. doi: 10.1007/s00394-017-1396-0

Okuyama, T., Inoue, H., Ookuma, S., Satoh, T., Kano, K., Honjoh, S., et al. (2010). The ERK-MAPK pathway regulates longevity through SKN-1 and insulinlike signaling in Caenorhabditis elegans. J. Biol. Chem. 285, 30274-30281. doi: $10.1074 / j b c . M 110.146274$

Papaevgeniou, N., and Chondrogianni, N. (2018). Anti-aging and anti-aggregation properties of polyphenolic compounds in C. elegans. Curr. Pharm. Des. 24, 2107-2120. doi: 10.2174/1381612824666180515145652

Park, S., Artan, M., Han, S. H., Park, H. H., Jung, Y., Hwang, A. B., et al. (2020). VRK-1 extends life span by activation of AMPK via phosphorylation. Sci. Adv. 6:eaaw7824. doi: 10.1126/sciadv.aaw7824

Peixoto, H., Roxo, M., Krstin, S., Rohrig, T., Richling, E., and Wink, M. (2016). An Anthocyanin-rich extract of acai (Euterpe precatoria Mart.) increases stress resistance and retards aging-related markers in Caenorhabditis elegans. J. Agric. Food Chem. 64, 1283-1290. doi: 10.1021/acs.jafc.5b05812

Peng, C., Chan, H. Y., Li, Y. M., Huang, Y., and Chen, Z. Y. (2009). Black tea theaflavins extend the lifespan of fruit flies. Exp. Gerontol. 44, 773-783. doi: 10.1016/j.exger.2009.09.004

Perez-Vizcaino, F., and Duarte, J. (2010). Flavonols and cardiovascular disease. Mol. Aspects Med. 31, 478-494. doi: 10.1016/j.mam.2010.09.002

Pietsch, K., Saul, N., Menzel, R., Sturzenbaum, S. R., and Steinberg, C. E. (2009). Quercetin mediated lifespan extension in Caenorhabditis elegans is modulated by age-1, daf-2, sek-1 and unc-43. Biogerontology 10, 565-578. doi: 10.1007/s10522-008-9199-6

Pietsch, K., Saul, N., Swain, S. C., Menzel, R., Steinberg, C. E., and Sturzenbaum, S. R. (2012). Meta-analysis of global transcriptomics suggests that conserved genetic pathways are responsible for quercetin and tannic acid mediated longevity in C. elegans. Front. Genet. 3:48. doi: 10.3389/fgene.2012.00048

Proshkina, E., Lashmanova, E., Dobrovolskaya, E., Zemskaya, N., Kudryavtseva, A., Shaposhnikov, M., et al. (2016). Geroprotective and radioprotective activity of quercetin, (-)-epicatechin, and ibuprofen in Drosophila melanogaster. Front. Pharmacol. 7:505. doi: 10.3389/fphar.2016.00505

Qu, Z., Ji, S., and Zheng, S. (2020a). BRAF controls the effects of metformin on neuroblast cell divisions in C. elegans. Int. J. Mol. Sci. 22:178. doi: 10.1101/2020.11.03.367557

Qu, Z., Ji, S., and Zheng, S. (2020b). Glucose and cholesterol induce abnormal cell divisions via DAF-12 and MPK-1 in C. elegans. Aging 12, 16255-16269. doi: 10.18632/aging.103647
Ruderman, N. B., Xu, X. J., Nelson, L., Cacicedo, J. M., Saha, A. K., Lan, F., et al. (2010). AMPK and SIRT1: a long-standing partnership? Am. J. Physiol. Endocrinol. Metab. 298, E751-E760. doi: 10.1152/ajpendo.00745.2009

Sahin, E., Colla, S., Liesa, M., Moslehi, J., Muller, F. L., Guo, M., et al. (2011). Telomere dysfunction induces metabolic and mitochondrial compromise. Nature 470, 359-365. doi: 10.1038/nature09787

Salehi, B., Mishra, A. P., Nigam, M., Sener, B., Kilic, M., Sharifi-Rad, M., et al. (2018). Resveratrol: a double-edged sword in health benefits. Biomedicines 6:91. doi: 10.3390/biomedicines6030091

Salminen, A., and Kaarniranta, K. (2012). AMP-activated protein kinase (AMPK) controls the aging process via an integrated signaling network. Ageing Res. Rev. 11, 230-241. doi: 10.1016/j.arr.2011.12.005

Salminen, A., Ojala, J., Kaarniranta, K., and Kauppinen, A. (2012). Mitochondrial dysfunction and oxidative stress activate inflammasomes: impact on the aging process and age-related diseases. Cell Mol. Life Sci. 69, 2999-3013. doi: 10.1007/s00018-012-0962-0

Saul, N., Pietsch, K., Menzel, R., Sturzenbaum, S. R., and Steinberg, C. E. (2010). The longevity effect of tannic acid in Caenorhabditis elegans: disposable Soma meets hormesis. J. Gerontol. A Biol. Sci. Med. Sci. 65, 626-635. doi: 10.1093/gerona/glq051

Saul, N., Pietsch, K., Sturzenbaum, S. R., Menzel, R., and Steinberg, C. E. (2011). Diversity of polyphenol action in Caenorhabditis elegans: between toxicity and longevity. J. Nat. Prod. 74, 1713-1720. doi: 10.1021/np200011a

Seo, H. W., Cheon, S. M., Lee, M. H., Kim, H. J., Jeon, H., and Cha, D. S. (2015). Catalpol modulates lifespan via DAF-16/FOXO and SKN-1/Nrf2 activation in Caenorhabditis elegans. Evid. Based Complement. Alternat. Med. 2015:524878. doi: $10.1155 / 2015 / 524878$

Smith, M. R., Syed, A., Lukacsovich, T., Purcell, J., Barbaro, B. A., Worthge, S. A., et al. (2014). A potent and selective Sirtuin 1 inhibitor alleviates pathology in multiple animal and cell models of Huntington's disease. Hum. Mol. Genet. 23 , 2995-3007. doi: 10.1093/hmg/ddu010

Sobeh, M., Hamza, M. S., Ashour, M. L., Elkhatieb, M., El Raey, M. A., AbdelNaim, A. B., et al. (2020). A polyphenol-rich fraction from eugenia uniflora exhibits antioxidant and hepatoprotective activities in vivo. Pharmaceuticals 13:84. doi: $10.3390 / \mathrm{ph} 13050084$

Sohal, R. S., and Orr, W. C. (2012). The redox stress hypothesis of aging. Free Radic. Biol. Med. 52, 539-555. doi: 10.1016/j.freeradbiomed.2011.10.445

Song, B., Wang, H., Xia, W., Zheng, B., Li, T., and Liu, R. H. (2020a). Combination of apple peel and blueberry extracts synergistically induced lifespan extension via DAF-16 in Caenorhabditis elegans. Food Funct. 11, 6170-6185. doi: 10.1039/D0FO00718H

Song, B. B., Zheng, B. S., Li, T., and Liu, R. H. (2020b). SKN-1 is involved in combination of apple peels and blueberry extracts synergistically protecting against oxidative stress in Caenorhabditis elegans. Food Funct. 11, 5409-5419. doi: 10.1039/D0FO00891E

Strayer, A., Wu, Z., Christen, Y., Link, C. D., and Luo, Y. (2003). Expression of the small heat-shock protein Hsp16-2 in Caenorhabditis elegans is suppressed by Ginkgo biloba extract EGb 761. FASEB J. 17, 2305-2307. doi: 10.1096/fj.03-0376fje

$\mathrm{Su}$, S., and Wink, M. (2015). Natural lignans from Arctium lappa as antiaging agents in Caenorhabditis elegans. Phytochemistry 117, 340-350. doi: 10.1016/j.phytochem.2015.06.021

Sugawara, T., and Sakamoto, K. (2020). Quercetin enhances motility in aged and heat-stressed Caenorhabditis elegans nematodes by modulating both HSF-1 activity, and insulin-like and p38-MAPK signalling. PLOS ONE 15:e0238528. doi: 10.1371 /journal.pone. 0238528

Swindell, W. R. (2009). Heat shock proteins in long-lived worms and mice with insulin/insulin-like signaling mutations. Aging 1, 573-577. doi: 10.18632/aging. 100058

Tambara, A. L., de Los Santos Moraes, L., Dal Forno, A. H., Boldori, J. R., Goncalves Soares, A. T., de Freitas Rodrigues, C., et al. (2018). Purple pitanga fruit (Eugenia uniflora L.) protects against oxidative stress and increase the lifespan in Caenorhabditis elegans via the DAF-16/FOXO pathway. Food Chem. Toxicol. 120, 639-650. doi: 10.1016/j.fct.2018.07.057

Troemel, E. R., Chu, S. W., Reinke, V., Lee, S. S., Ausubel, F. M., and Kim, D. H. (2006). p38 MAPK regulates expression of immune response genes and contributes to longevity in C. elegans. PLoS Genet. 2:e183. doi: 10.1371/journal.pgen.0020183 
Tullet, J. M. (2015). DAF-16 target identification in C. elegans: past, present and future. Biogerontology 16, 221-234. doi: 10.1007/s10522-014-9527-y

Turgut, N. H., Mert, D. G., Kara, H., Egilmez, H. R., Arslanbas, E., Tepe, B., et al. (2016). Effect of black mulberry (Morus nigra) extract treatment on cognitive impairment and oxidative stress status of D-galactose-induced aging mice. Pharm. Biol. 54, 1052-1064. doi: 10.3109/13880209.2015.1101476

Wallace, T. C., and Giusti, M. M. (2015). Anthocyanins. Adv. Nutr. 6, 620-622. doi: 10.3945/an.115.009233

Wang, H., Liu, J., Li, T., and Liu, R. H. (2018). Blueberry extract promotes longevity and stress tolerance via DAF-16 in Caenorhabditis elegans. Food Funct. 9, 5273-5282. doi: 10.1039/C8FO01680A

Wang, X., Zhang, J. L., Lu, L. L., and Zhou, L. J. (2015). The longevity effect of echinacoside in Caenorhabditis elegans mediated through daf-16. Biosci. Biotechnol. Biochem. 79, 1676-1683. doi: 10.1080/09168451.2015.1046364

Wilson, M. A., Shukitt-Hale, B., Kalt, W., Ingram, D. K., Joseph, J. A., and Wolkow, C. A. (2006). Blueberry polyphenols increase lifespan and thermotolerance in Caenorhabditis elegans. Aging Cell 5, 59-68. doi: 10.1111/j.1474-9726.2006.00192.x

Wood, J. G., Rogina, B., Lavu, S., Howitz, K., Helfand, S. L., Tatar, M., et al. (2004). Sirtuin activators mimic caloric restriction and delay ageing in metazoans. Nature 430, 686-689. doi: 10.1038/nature02789

Wu, X., Al-Amin, M., Zhao, C., An, F., Wang, Y., Huang, Q., et al. (2020). Catechinic acid, a natural polyphenol compound, extends the lifespan of Caenorhabditis elegans via mitophagy pathways. Food Funct. 11, 5621-5634. doi: 10.1039/D0FO00694G

Xiong, L. G., Chen, Y. J., Tong, J. W., Gong, Y. S., Huang, J. A., and Liu, Z. H. (2018). Epigallocatechin-3-gallate promotes healthy lifespan through mitohormesis during early-to-mid adulthood in Caenorhabditis elegans. Redox Biol. 14, 305-315. doi: 10.1016/j.redox.2017.09.019

Xiong, L. G., Huang, J. A., Li, J., Yu, P. H., Xiong, Z., Zhang, J. W., et al. (2014). Black tea increased survival of Caenorhabditis elegans under stress. J. Agric. Food Chem. 62, 11163-11169. doi: 10.1021/jf503120j

Yaguchi, Y., Komura, T., Kashima, N., Tamura, M., Kage-Nakadai, E., Saeki, S., et al. (2014). Influence of oral supplementation with sesamin on longevity of Caenorhabditis elegans and the host defense. Eur. J. Nutr. 53, 1659-1668. doi: 10.1007/s00394-014-0671-6

Yan, F. J., Chen, Y. S., Azat, R., and Zheng, X. D. (2017). Mulberry anthocyanin extract ameliorates oxidative damage in HepG2 cells and prolongs the lifespan of Caenorhabditis elegans through MAPK and Nrf2 pathways. Oxid. Med. Cell. Longev. 2017:7956158. doi: 10.1155/2017/7 956158

Ye, Y., Gu, Q., and Sun, X. (2020). Potential of Caenorhabditis elegans as an antiaging evaluation model for dietary phytochemicals: a review.
Compr. Rev. Food Sci. Food Saf. 19, 3084-3105. doi: 10.1111/1541-4337. 12654

Yoon, D. S., Cha, D. S., Choi, Y., Lee, J. W., and Lee, M. H. (2019). MPK$1 /$ ERK is required for the full activity of resveratrol in extended lifespan and reproduction. Aging Cell 18:e12867. doi: 10.1111/acel.12867

Yoon, D. S., Choi, Y., Choi, S. M., Park, K. H., and Lee, J. W. (2015). Different effects of resveratrol on early and late passage mesenchymal stem cells through beta-catenin regulation. Biochem. Biophys. Res. Commun. 467, 1026-1032. doi: 10.1016/j.bbrc.2015.10.017

Yue, Y., Shen, P., Xu, Y., and Park, Y. (2019). p-Coumaric acid improves oxidative and osmosis stress responses in Caenorhabditis elegans. J. Sci. Food Agric. 99, 1190-1197. doi: 10.1002/jsfa.9288

Zarse, K., Jabin, S., and Ristow, M. (2012). L-Theanine extends lifespan of adult Caenorhabditis elegans. Eur. J. Nutr. 51, 765-768. doi: 10.1007/s00394-012-0341-5

Zhang, J. (2006). Resveratrol inhibits insulin responses in a SirT1-independent pathway. Biochem. J. 397, 519-527. doi: 10.1042/BJ20050977

Zhang, L., Jie, G., Zhang, J., and Zhao, B. (2009). Significant longevity-extending effects of EGCG on Caenorhabditis elegans under stress. Free Radic. Biol. Med. 46, 414-421. doi: 10.1016/j.freeradbiomed.2008.10.041

Zhao, L., Zhao, Y., Liu, R. H., Zheng, X. N., Zhang, M., Guo, H. Y., et al. (2017). The transcription factor DAF-16 is essential for increased longevity in C. elegans exposed to Bifidobacterium longum BB68. Sci. Rep. 7:7408. doi: 10.1038/s41598-017-07974-3

Zheng, S., Qu, Z., Zanetti, M., Lam, B., and Chin-Sang, I. (2018). C. elegans PTEN and AMPK block neuroblast divisions by inhibiting a BMP-insulin-PP2AMAPK pathway. Development 145:dev166876. doi: 10.1242/dev.166876

Zheng, S. Q., Huang, X. B., Xing, T. K., Ding, A. J., Wu, G. S., and Luo, H. R. (2017). Chlorogenic acid extends the lifespan of Caenorhabditis elegans via insulin/IGF-1 signaling pathway. J. Gerontol. A Biol. Sci. Med. Sci. 72, 464-472. doi: $10.1093 /$ gerona/glw105

Conflict of Interest: The authors declare that the research was conducted in the absence of any commercial or financial relationships that could be construed as a potential conflict of interest.

Copyright (c) 2021 Liu, Guo, Wang, Zheng, Qu and Liu. This is an open-access article distributed under the terms of the Creative Commons Attribution License (CC $B Y)$. The use, distribution or reproduction in other forums is permitted, provided the original author(s) and the copyright owner(s) are credited and that the original publication in this journal is cited, in accordance with accepted academic practice. No use, distribution or reproduction is permitted which does not comply with these terms. 